\section{Vulnerabilidad social en mujeres embarazadas de una comuna de la Región Metropolitana}

\author{
ESTELA ARCOS ${ }^{1, a}$, LUZ ANGÉLICA MUÑOZ ${ }^{2, b}$, \\ XIMENA SÁNCHEZ ${ }^{3, c}$, ANTONIA VOLLRATH ${ }^{1, \mathrm{~d}}$, CARLA LATORRE $^{1, \mathrm{e}}$, \\ CARLA BONATTI ${ }^{1, \mathrm{e}}$, JOSSELINE JAUREGUI ${ }^{4, \mathrm{f}}$
}

\section{Vulnerability of pregnant women living in a community of Metropolitan Santiago}

Background: The program "Chile grows with you" is a part of the Chilean social protection system oriented to decrease social inequities from pregnancy (a socially secure pregnancy) to four years of age, directed to the poorest $40 \%$ of the population. Aim: To determine the incidence of social vulnerability and its determinants, starting at the gestation period. Material and Methods: Data was obtained from anonymous secondary data based on the records of the first assessment of pregnant women and from social protection surveys, provided by the Ministry of Planning and Cooperation. Results: The incidence of social vulnerability was high. However there was a disparity between the figures obtained from the social protection records and the survey carried out during the first assessment of pregnant women (91 and $27 \%$ respectively). The psychosocial risk was higher among vulnerable women (42\% compared to $28 \%$ among women not considered vulnerable). This risk was associated with lack of family support, depressive symptoms, gender violence, substance abuse and maternity conflicts. Working conditions were precarious with a low level of social security, there were habitability problems, disability, dependency, female householders and a mean income below the threshold of poverty. Conclusions: Among vulnerable families, there are adverse determinants that attempt against a socially secure pregnancy and integral development of children.

(Rev Med Chile 2011; 139: 739-747).

Key words: Pregnancy; Public policy; Social control policies.

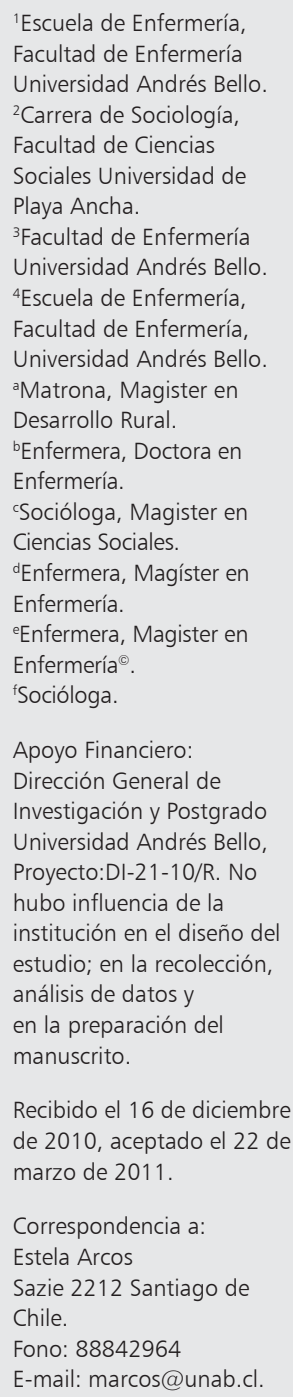

Recibido el 16 de diciembre de 2010, aceptado el 22 de marzo de 2011.

Correspondencia a: Estela Arcos

Sazie 2212 Santiago de

Chile.

Fono: 88842964

E-mail:marcos@unab.cl.

L os procesos de reforma del sistema de salud en Chile de la década 1990-99, consideraron Jla implementación de un modelo basado en el enfoque de salud familiar; para que los equipos de salud asumieran la responsabilidad del cuidado de la salud de las personas, familias y comunidades otorgando atención integral y alta capacidad resolutiva. Esta reforma supuso un cambio profundo, que implicó un replanteamiento tanto en los objetivos y metas del sistema como en las formas de llevarlas a cabo.

Los desafíos asumidos por la política social, para el período 2006-2010, fueron transitar desde un sistema de protección social a partir de las condiciones mínimas de vida hacia otra de derechos sociales garantizados y participación ciudadana ${ }^{1}$. En términos de infancia vulnerable, se ha señalado que invertir en los primeros años de vida es una de las medidas que con más probabilidad permitiría reducir las desigualdades en el lapso de una generación, porque las experiencias vividas en la primera infancia (entre el desarrollo del feto y los ocho años de edad) y adolescencia proporcionan las bases sobre las que se sustentará el resto del desarrollo vital.

Chile tiene indicadores de mortalidad materna 
e infantil y de cobertura en atención de salud que lo ubican entre países con una posición sanitaria adelantada ${ }^{2}$, aun cuando persisten profundas desigualdades e inequidades en la sociedad cuya evidencia es la invariabilidad del indicador de desigualdad ${ }^{3}$. Estas inequidades se traducen en una condición de vulnerabilidad que afecta las trayectorias de vida de las personas, en términos de posibilidades y oportunidades ${ }^{4-7}$. En el año 2009, el ingreso autónomo de 10\% de los hogares con mayores ingresos era 46 veces más grande que el ingreso autónomo de $10 \%$ de los hogares con menores ingresos. Este fenómeno no es capturado totalmente por el índice de desigualdad, debido a que éste pondera mayormente lo que sucede en la parte central de la distribución y no en los extremos ${ }^{6-8}$.

La vulnerabilidad se relaciona con las características biológicas y psicológicas de las personas, con las condiciones sociales y ambientales, el ciclo vital, la estructura y funcionalidad de la familia y el territorio donde viven, porque la pobreza se concentra en unidades vecinales territoriales generando espacios de vulnerabilidad y exclusión social que implican fragilidad, amenaza y susceptibilidad a daños en la salud ${ }^{9-13}$.

En contextos vulnerables la maternidad "segura" enfrenta condiciones de especial fragilidad, ya que existen evidencias que demuestran la asociación entre vulnerabilidad social con mayores niveles de estrés y ansiedad de la madre antes y durante el embarazo y, como consecuencia, mayor incidencia de prematuridad, bajo peso al nacer, destete precoz, deficiente calidad del cuidado del niño, mayores tasas de rezago, retraso del desarrollo infantil, déficit atencional e hiperactividad, problemas del lenguaje, deficiente competencia social y el comportamiento del individuo para toda la vida ${ }^{14-20}$.

La Red Protege contiene, entre otros, el programa Chile Crece Contigo (PChCC) que es una propuesta pública sistémica orientada a disminuir desigualdades desde la gestación (maternidad socialmente segura) hasta los cuatro años de edad, especialmente, en personas de $40 \%$ más pobre ${ }^{21}$. El argumento central del programa es equidad desde el principio, porque las evidencias indican que los primeros años de desarrollo humano determinan el comportamiento de la salud y el aprendizaje para toda la vida $a^{3,14,15}$.

Del debate sobre política de protección social, surgió la necesidad de investigar a quiénes apoya la Red Protege y cuál es su efectividad en la entrega de prestaciones de la oferta pública a la población beneficiaria, especialmente, en zonas geográficas con alta vulnerabilidad social ${ }^{12,13,21}$. Como primera etapa, se presenta un análisis descriptivo del contexto social de 1.656 gestantes y sus familias, adscritas al PChCC en el año 2009 en la comuna de Pudahuel, con el fin de determinar la incidencia de la vulnerabilidad social y tipificar los determinantes sociales que, desde la gestación, marcan la necesidad de protección social para las gestante y sus familias.

\section{Material y Método}

Las fuentes analizadas fueron bases de datos innominados sobre registros de: i) la encuesta "Primer control de la gestante", aplicada por una matrona durante el ingreso al control prenatal a 1.656 gestantes que realizaron su control prenatal en centros de la red pública de salud de la comuna de Pudahuel, y ii) la Ficha de Protección Social (FPS) aplicada al jefe de hogar (JH), por funcionarios del Departamento de Estratificación Social de la Ilustre Municipalidad de Pudahuel. Ambas bases fueron entregadas empalmadas por la Subsecretaría del Ministerio de Planificación (MIDEPLAN). Se garantizó el secreto estadístico y confidencialidad de los datos a través de la formalización institucional del proyecto, tanto con la Corporación Municipal como con MIDEPLAN. La investigación contó con la certificación del Comité de Ética de la Facultad de Enfermería de la Universidad Andrés Bello.

De la encuesta "Primer control de la gestante" se utilizó información relacionada con escolaridad, presencia y tipo de discapacidad, edad gestacional al ingreso control prenatal, aplicación de pauta psicosocial, condición y causa del riesgo psicosocial, percepción de vulnerabilidad social por la profesional que aplicó la encuesta, aceptación de visita domiciliaria y persona que acompaña a la embarazada al primer control. Respecto a la FPS, se utilizó la información sobre puntaje alcanzado, perfil del jefe de hogar, capacidad generadora de ingresos de la familia, tamaño y estructura de la familia, condiciones de habitabilidad de la vivienda y acceso a servicios básicos. La validación de los instrumentos fue realizada por la División Social de MIDEPLAN. 
Vulnerabilidad social en mujeres embarazadas - E. Arcos et al

Para cumplir con el objetivo del estudio, se re-codificó y re-etiquetó las variables relacionadas con vulnerabilidad social: edad, educación, riesgo psicosocial, acompañante de la gestante al control, ingreso JH y familia, tamaño de la familia, personas independientes y dependientes, tasa de dependencia y precariedad laboral.

En una primera etapa, se determinó la incidencia de vulnerabilidad social (VS) en 1.656 familias con un análisis comparativo entre familias con y sin VS y, en una segunda etapa se identificaron las mujeres embarazadas (1.656) del PChCC, las cuales fueron caracterizadas según VS de las familias de pertenencia. Se realizó una depuración de la fuente de datos bruta en función de la proporción de datos omitidos de cada variable, eliminándose aquellas que tenían más de $30 \%$ de datos faltantes.

Para establecer la incidencia VS se utilizó el criterio de MIDEPLAN sobre el puntaje de FPS que define la presencia o ausencia de la condición. Las familias eran vulnerables cuando alcanzaron menor o igual a 11.734 puntos (40\% más pobre), considerándose como "pobreza dura” los núcleos con puntajes menores o igual a ( $\leq 4.213$ puntos). El análisis estadístico se realizó a través de índices, estadígrafos descriptivos de posición y dispersión y análisis de razones, tasas, utilizándose programa computacional estadístico SPSS.

\section{Resultados}

En las familias estudiadas, la magnitud de VS fue alta $(91,4 \%), 1.514$ familias lograron puntaje inferior a 11.735 puntos en la FPS, Figura 1. En la población con VS, 779 familias $(51,4 \%)$ presen- taron la condición de "pobreza dura", menos de 4.213 puntos en la FPS. Se confirmaron disparidades sustantivas entre la detección de vulnerabilidad por aplicación de la FPS y por percepción del profesional matrona que aplicó la encuesta del Primer Control de la Gestante (91,4\% vs 26,6), Figura 2.

Respecto al perfil sociodemográfico, Tabla 1 , alrededor de $70 \%$ de las gestantes se embarazó en una edad biológicamente segura, entre los 20 y 34 años, observándose una mayor presencia de gestantes adolescentes en el grupo vulnerable. La escolaridad fue menor en gestantes vulnerables y la ascendencia de pueblos originarios fue más alta en gestantes no vulnerables.

Las familias vulnerables tenían 30\% más de madres jefas de hogar que las no vulnerables.

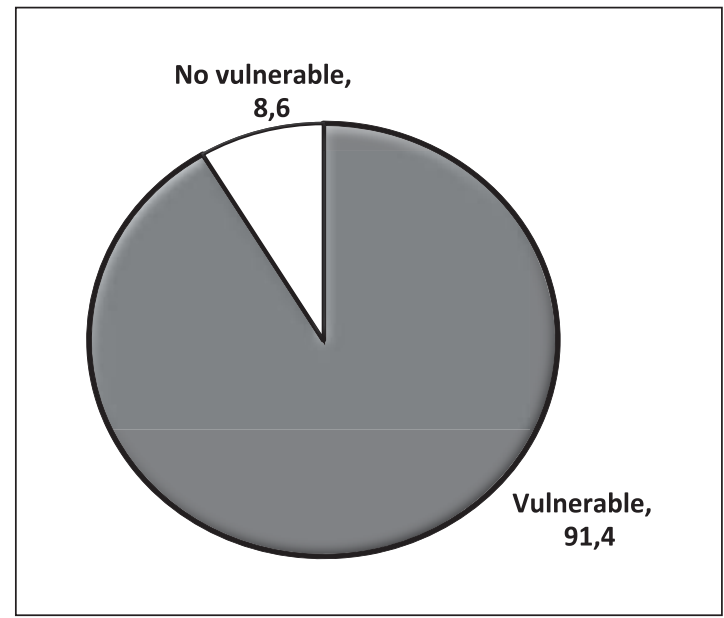

Figura 1. Vulnerabilidad de 1.656 gestantes/familias, según puntaje logrado en Ficha de Protección social, Comuna de Pudahuel, año 2009.

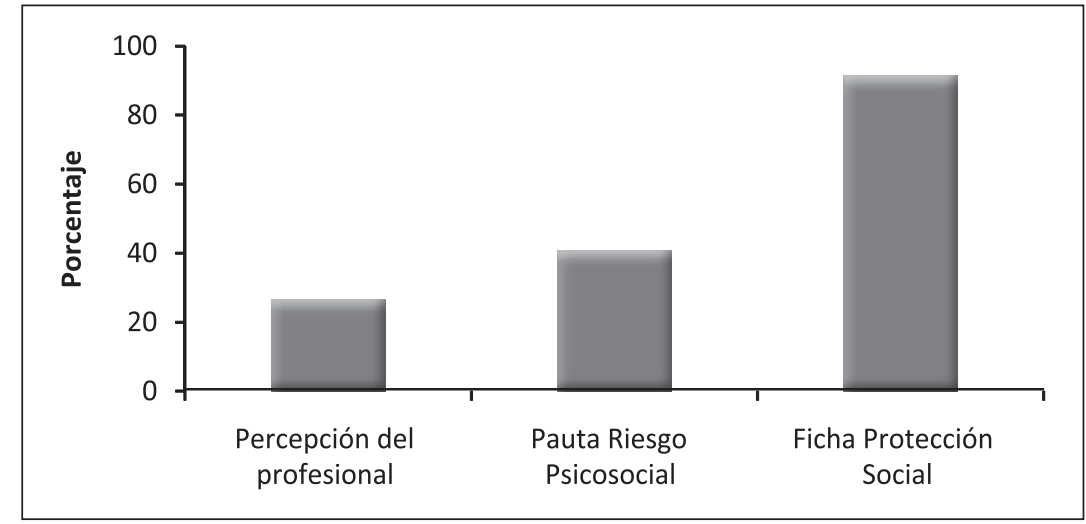

Figura 2. Disparidades porcentuales en la detección de vulnerabilidad y riesgo psicosocial en 1.656 mujeres-familias, según método utilizado. 
Tabla 1. Perfil sociodemográfico de las gestantes adscritas al programa Chile Crece Contigo en la comuna de Pudahuel, según vulnerabilidad social por puntaje FPS, año 2009

\begin{tabular}{|c|c|c|c|c|c|}
\hline \multirow[t]{2}{*}{ Variable } & \multirow[t]{2}{*}{$\begin{array}{l}\text { Rango/ } \\
\text { categoría }\end{array}$} & \multicolumn{2}{|c|}{$\begin{array}{c}\text { Vulnerable } \\
(1.514)\end{array}$} & \multicolumn{2}{|c|}{$\begin{array}{c}\text { No vulnerable } \\
(142)\end{array}$} \\
\hline & & $\mathbf{n}$ & $\%$ & $\mathbf{n}$ & $\%$ \\
\hline \multirow[t]{3}{*}{ Edad } & 14 - 19 años & 261 & 17,2 & 19 & 13,4 \\
\hline & 20 - 34 años & 1.042 & 68,9 & 99 & 69,7 \\
\hline & 35 - 45 años & 211 & 13,9 & 24 & 16,9 \\
\hline \multirow{3}{*}{$\begin{array}{l}\text { Años de escolaridad } \\
\text { aprobados }\end{array}$} & 0 a 8 años & 307 & 20,3 & 14 & 9,9 \\
\hline & 9 a 11 años & 392 & 25,9 & 26 & 18,3 \\
\hline & $\geq 12$ años & 815 & 53,9 & 102 & 71,8 \\
\hline $\begin{array}{l}\text { Ascendencia pueblos } \\
\text { originarios }\end{array}$ & Sí & 195 & 18,8 & 25 & 28,4 \\
\hline Trabajo remunerado & Sí & 548 & 50,5 & 48 & 46,6 \\
\hline Seguridad social & Sí & 170 & 31,0 & 38 & 79,2 \\
\hline Riesgo psicosocial & Sí & 636 & 42,0 & 40 & 28,2 \\
\hline
\end{tabular}

Tabla 2. Causas de riesgo reportadas por las gestantes en riesgo psicosocial adscritas al PChCC en la Comuna de Pudahuel, según vulnerabilidad social por puntaje FPS, año 2009

\begin{tabular}{|lccccc|}
\hline & & \multicolumn{5}{c|}{ Riesgo Psicosocial } \\
\hline Variable & $\begin{array}{c}\text { Rango/ } \\
\text { Categoría }\end{array}$ & $\begin{array}{c}\text { Vulnerable } \\
\text { (636) }\end{array}$ & $\begin{array}{c}\text { No vulnerable } \\
\text { (40) }\end{array}$ \\
\hline $\begin{array}{l}\text { Insuficiente apoyo } \\
\text { familiar }\end{array}$ & Sí & 234 & 36,8 & 17 & 42,5 \\
\hline Síntomas depresivos & Sí & 219 & 34,4 & 20 & 50,0 \\
\hline Violencia de género & Sí & 171 & 26,9 & 17 & 42,5 \\
\hline Abuso de sustancias & Sí & 160 & 25,2 & 9 & 22,5 \\
\hline $\begin{array}{l}\text { Conflictos con la ma- } \\
\text { ternidad }\end{array}$ & Sí & 111 & 17,5 & 6 & 15,0 \\
\hline Otros riesgos & & & & & \\
\hline
\end{tabular}

Sobre la situación ocupacional de las mujeres, no se observaron diferencias sustanciales en la proporción de madres que trabajaban entre los grupos. Sin embargo, en el grupo vulnerable más de $60 \%$ trabajaba sin contrato y $69 \%$ lo hacía sin sistema previsional, Tabla 1.

La presencia de riesgo psicosocial fue mayor en mujeres con VS (42,0\% versus $28,2 \%$ ), siendo los más referidos el insuficiente apoyo familiar, los síntomas depresivos, violencia de género, abuso de substancias y conflictos con la maternidad. Las embarazadas adolescentes reportaron falta de apoyo del progenitor, Tabla 2.

En el caso de las familias de gestantes vulnerables los ingresos que percibe el $\mathrm{JH}$ por trabajo apenas representan $62 \%$ del ingreso del $\mathrm{JH}$ de familias no vulnerables. Asimismo, al considerar el sexo mujer del jefe de hogar se encontraron bajas sustanciales en los ingresos para todos los grupos, observándose las mayores diferencias en los grupos no vulnerables, Tabla 3. Al considerar el ingreso per cápita de las familias, las diferencias económicas aumentan, ya que las familias vulnerables presentan un mayor número de integrantes con dependencia económica -las familias vulnerables presentan una mayor cantidad de allegados y niños-situación que disminuye los ingresos del núcleo, Tablas 3 y 4.

En familias vulnerables, las condiciones de habitabilidad eran estructuralmente más precarias, residían en viviendas y sitios cedidos (58,7\% y $60,3 \%$ respectivamente). Una de cada dos familias compartían la vivienda $y$, en 2 de cada tres 
Vulnerabilidad social en mujeres embarazadas - E. Arcos et al

Tabla 3. Capacidad generadora de ingresos de la familia de gestantes adscritas al PChCC en la Comuna de Pudahuel, según vulnerabilidad social por FPS, año 2009

\begin{tabular}{|c|c|c|c|c|c|}
\hline \multirow[t]{2}{*}{ Variable } & \multirow[t]{2}{*}{ Rango/categoría } & \multicolumn{2}{|c|}{ Vulnerable } & \multicolumn{2}{|c|}{ No vulnerable } \\
\hline & & $\mathbf{n}$ & $\%$ & $\mathbf{n}$ & $\%$ \\
\hline Personas en edad de trabajar por familia (de 16 a 65 años) & Con trabajo & 1.574 & 68,9 & 200 & 68,0 \\
\hline \multirow[t]{2}{*}{ Fuerza laboral } & Activa & 3.265 & 57,6 & 370 & 82,4 \\
\hline & Inactiva & 2.406 & 42,4 & 76 & 16,6 \\
\hline \multirow[t]{3}{*}{ Jefe de hogar $(J H)$, según sexo } & Mujer & 1.044 & 69,0 & 59 & 41,5 \\
\hline & Hombre & 470 & 31,0 & 83 & 58,5 \\
\hline & & $(1.515)$ & & $(142)$ & \\
\hline \multirow[t]{3}{*}{ Proporción de ocupación del JH, según sexo } & Hombre con trabajo & 398 & 40,6 & 74 & 66,7 \\
\hline & Mujer con trabajo & 582 & 59,4 & 37 & 33,3 \\
\hline & & $(980)$ & & $(111)$ & \\
\hline \multirow[t]{3}{*}{ Tipo de ocupación JH que trabaja } & Permanente & 923 & 94,2 & 107 & 96,4 \\
\hline & Ocasional & 57 & 5,8 & 4 & 3,6 \\
\hline & & $(980)$ & & $(111)$ & \\
\hline Seguridad social JH & Sí & 409 & 28,5 & 94 & 68,6 \\
\hline \multirow[t]{3}{*}{ Ingresos JH, por trabajo } & Media $\pm \mathrm{DE}$ & & & & \\
\hline & Hombre & \multicolumn{2}{|c|}{$\$ 146.369$} & \multicolumn{2}{|c|}{$\$ 228.500$} \\
\hline & Mujer & \multicolumn{2}{|c|}{$\$ 65.609$} & \multicolumn{2}{|c|}{$\$ 111.506$} \\
\hline Ingreso per cápita & Media $\pm D E$ & \multicolumn{2}{|c|}{$\$ 41.498$} & \multicolumn{2}{|c|}{$\$ 100.848$} \\
\hline Precariedad laboral ${ }^{(1)}$ & Sí & 549 & 56,0 & 10 & 9,0 \\
\hline
\end{tabular}

(1)Para el caso de esta investigación la precariedad laboral fue entendida como la asociación de ingresos por trabajo inferior a 165.000 (sueldo mínimo) y ausencia de seguridad laboral por falta de previsión. Así mismo, el porcentaje de precariedad laboral fue calculado sobre el total de trabajadores registrados en cada grupo de vulnerabilidad.

viviendas el baño no era de uso exclusivo de la familia, Tabla 5.

En suma, las familias vulnerables de las gestantes mostraron una situación más adversa respecto a riesgo psicosocial, discapacidad, hacinamiento, relación de dependencia, seguridad social, condiciones de habitabilidad y precariedad laboral y económica.

\section{Discusión}

El análisis de datos secundarios sobre el PChCC y la FPS permitió visibilizar la alta incidencia de vulnerabilidad de las familias de mujeres embarazadas $(91,4 \%)$, con una proporción indicadora de pobreza dura e inequidad social en $51,4 \%$, lo que según los criterios de MIDEPLAN les garantizó el acceso al Programa Puente y Chile Solidario. Acorde a la literatura revisada, se trata de una vulnerabilidad socio-política que se deriva de la pertenencia a un grupo, género, territorio, medio, condición socio-económica, cultura y ambiente que expone a las personas a situaciones de falta de poder, autonomía y desprotección social ${ }^{9,22}$.

En territorios muy vulnerables se naturaliza la vulnerabilidad social, hecho que por sí mismo explica las diferencias porcentuales observadas entre VS por percepción de la profesional de salud y por FPS (26,6\% vs $91,4 \%)$, situación que invisibiliza a muchas personas que tienen una alta susceptibilidad a daños en la salud ${ }^{10}$. Este hecho es 
Tabla 4. Características de la familia de gestantes adscritas al PChCC según vulnerabilidad social por FPS, comuna de Pudahuel, año 2009

\begin{tabular}{|c|c|c|c|c|c|}
\hline \multirow{2}{*}{ Variable } & \multirow[t]{2}{*}{ Rango/categoría } & \multicolumn{2}{|c|}{ Vulnerable } & \multicolumn{2}{|c|}{ No vulnerable } \\
\hline & & $\mathbf{n}$ & $\%$ & & $\%$ \\
\hline \multirow[t]{3}{*}{ Tamaño (personas) } & 1 a 4 & 1.099 & 72,6 & 124 & 87,3 \\
\hline & 5 y más & 415 & 27,4 & 18 & 12,7 \\
\hline & Total & 1.514 & 100,0 & 142 & 100,0 \\
\hline \multirow[t]{4}{*}{ Estructura (edad) } & $<18$ años & 2.561 & 45,2 & 89 & 19,8 \\
\hline & 18 a 64 años & 3.025 & 53,4 & 335 & 79,1 \\
\hline & $\geq 65$ años & 84 & 1,5 & 5 & 1,1 \\
\hline & Total & 5.670 & 100,0 & 449 & 100,0 \\
\hline \multirow[t]{5}{*}{ Parentezco con JH } & Jefe Hogar & 1.514 & 26,7 & 142 & 31,6 \\
\hline & Cónyuge & 752 & 13,3 & 110 & 24,5 \\
\hline & Hija & 2.810 & 49,5 & 172 & 38,3 \\
\hline & Otros & 594 & 10,5 & 25 & 5,6 \\
\hline & Total & 5.670 & 100,0 & 449 & 100,0 \\
\hline Discapacidad & $\mathrm{N}^{\circ}$ Personas & 253 & 16,7 & 17 & 12, \\
\hline
\end{tabular}

Tabla 5. Condiciones de la vivienda de las familias de gestantes adscritas al PChCC en la comuna de Pudahuel, según vulnerabilidad social por FPS, año 2009

\begin{tabular}{|c|c|c|c|c|c|}
\hline Variables vivienda & Rango/categoría & Vulr & ble & No v & rable \\
\hline & & & $\%$ & $n$ & $\%$ \\
\hline Tipo & Casa o Departamento & 1.219 & 80,5 & 121 & 85,2 \\
\hline & Mediagua / pieza / choza & 295 & 19,5 & 21 & 14,8 \\
\hline Tenencia vivienda & Propia & 419 & 27,7 & 64 & 45,1 \\
\hline & Arrendada & 169 & 11,2 & 19 & 13,4 \\
\hline & Cedida, uso gratuito & 913 & 60,3 & 59 & 41,5 \\
\hline & Ocupación irregular & 13 & 8,6 & 0 & \\
\hline Tenencia sitio & Propia & 396 & 26,1 & 61 & 43,0 \\
\hline & Arrendada & 169 & 11,2 & 19 & 13,4 \\
\hline & Cedida, uso gratuito & 936 & 61,8 & 62 & 43,7 \\
\hline & Ocupación irregular & 13 & 0,9 & 0 & 0,0 \\
\hline & & $(1.514)$ & & $(142)$ & \\
\hline Familia principal ocupante & Sí & 727 & 48,0 & 91 & 64,1 \\
\hline Obtención agua & Red pública con medidor propio & 1.070 & 70,7 & 109 & 76,8 \\
\hline & Red pública con medidor compartido & 431 & 27,3 & 32 & 22,5 \\
\hline & Otro & 13 & 8,6 & 1 & 0,7 \\
\hline El agua a la casa llega por & Llave dentro de la vivienda & 1.329 & 87,8 & 134 & 94,4 \\
\hline & Llave dentro del sitio & 164 & 10,8 & 7 & 4,9 \\
\hline & Acarreo & 21 & 1,4 & 1 & 0,7 \\
\hline Conexión sistema de baño & Alcantarillado & 1.347 & 89,0 & 131 & 92,3 \\
\hline & Fosa / letrina / cajón / pozo negro & 167 & 11,0 & 11 & 7,7 \\
\hline Exclusividad uso baño & Sí & 629 & 41,5 & 82 & 57,7 \\
\hline
\end{tabular}


de suma importancia, porque los equipos de salud planifican la oferta de prestaciones específicas a través de los datos que recogen y procesan, factor que podría ser una limitante para la efectividad de la entrega de prestaciones de la Red Protege.

Las tasas de jefaturas de JH femeninas observadas fueron similares a las reportadas por MIDEPLAN el año 2007 en Tarapacá $(45,3 \%)$ y Magallanes (43,1\%), regiones con las tasas más altas del país. Se ha señalado que hay una tendencia creciente de este fenómeno, sobre todo en familias monoparentales, extensas y compuestas ${ }^{23}$. También, los resultados muestran una alta proporción de embarazadas adolescentes que, al igual como lo señala Sunkel, está asociada a deserción escolar, crianza sin pareja y dependencia de la familia de origen $^{23}$.

En este estudio, la precariedad laboral de las mujeres que trabajaban demostró una fuerte presencia de trabajo informal, bajos ingresos y baja cobertura de seguridad social, situación que implica la ausencia de beneficios para el real ejercicio de una maternidad segura e infancia protegida. Se ha señalado que la salud reproductiva, neonatal, mental y familiar se deteriora cuando las mujeres tienen empleos precarios, con embarazos sin seguridad social, habitabilidad informal e insegura de la vivienda, baja escolaridad, riesgo psicosocial, ausencia de la pareja en el hogar y alta tasa de dependencia ${ }^{16,24}$.

La tasa de dependencia encontrada, confirma que una familia es más vulnerable cuando en el hogar hay embarazadas, lactantes o niños pequeños y estudiantes ${ }^{23}$. Además, la mujer JH que trabaja abarca una doble jornada laboral, combina el trabajo asalariado y el trabajo no remunerado en casa, lo que la expone a padecer altos niveles de estrés por la sobrecarga de género ${ }^{23,25-27}$. Se ha señalado que un sustrato sociocultural adverso predice resultados negativos en las trayectorias de vida en la adultez desde la vida intrauterina, neonatal y de la infancia ${ }^{14-16,24}$. Barker, citado por Talge, señaló que la enfermedad coronaria, diabetes tipo 2 , accidentes vasculares, hipertensión y mayores tasas de mortalidad en la adultez están asociadas con antecedentes de desnutrición fetal y bajo peso al nacer ${ }^{17}$.

Los resultados reportados muestran determinantes sociales potencialmente adversos para una maternidad segura y un desarrollo integral del infante, lo que amerita acciones efectivas de protección social con el fin de aminorar sus consecuencias negativas. Se ha señalado que, para avanzar en equidad desde el principio es perentorio realizar un trabajo intersectorial e interdisciplinario que refuerce las competencias parentales en la satisfacción de las necesidades evolutivas de la infancia a través de una distribución equitativa, justa y oportuna de las prestaciones de los programas sociales de la Red Protege $e^{14,28-30}$.

Para soluciones integrales y de alto impacto en maternidad socialmente segura, es fundamental que los profesionales de la red pública de salud, por una parte, refuercen el trabajo integrado con un enfoque de derechos humanos, determinantes sociales y promuevan la participación ciudadana para mejorar la efectividad de los programas sociales de la Red Protege ${ }^{9,14,15,21,31-37}$. Por otra, por la interacción cotidiana que tienen con mujeres, infantes y familias vulnerables, a través de las prestaciones universales contenidas en los programas de salud, deben asumir el desafío de liderar la vigilancia, monitoreo y evaluación de la efectividad de los programas sociales ${ }^{32,38,39}$.

Para llevarlo a cabo, los profesionales de la atención primaria de salud tienen que ampliar la información sobre la forma cómo los determinantes sociales y económicos afectan la salud de las personas que atienden, porque su conocimiento les permitirá, por una parte, proponer estrategias de promoción de salud pertinentes a su realidad territorial y, por otra, resolver la complejidad de los problemas de salud que enfrentan a través de una práctica sanitaria contextualizada que tiene un gran potencial en la disminución de brechas de equidad social y disparidades en salud d,14,24,30-32 $^{\text {. }}$ Para esto, requieren de un flujo permanentemente de información del sistema integrado de información social (SIIS), agregada y desagregada, para que midan la incidencia de la vulnerabilidad social de las familias y analicen sus consecuencias, tanto en su estructura y funcionalidad como en la distribución territorial.

Lo precedente, enfrenta dificultades evidentes por las limitaciones del SIIS para cumplir con el propósito mencionado, ya que las base de datos de MIDEPLAN del PChCC y FPS no tienen registros integrados. En función de lo expuesto, es posible deducir que el sistema de intercambio de datos del SIIS con otras instituciones públicas sea parcial, lo cual permite plantearnos algunas interrogantes sobre la pertinencia del diseño y funcionalidad del 
SIIS para monitorear la focalización de las prestaciones del PChCC y Red Protege a las mujeres, infantes y familias.

Las consecuencias de lo referido, se pueden traducir en fisuras y voces de alerta sobre la efectividad de dichos programas como soporte social a familias vulnerables. Es un hecho que si los equipos no cuentan con información de calidad, menos posibilidades tiene la ciudadanía de realizar un control ciudadano de aquello que desconoce ${ }^{40}$.

\section{Referencias}

1. Lavados C, Gajardo A. El principio de justicia y equidad en Chile. Acta bioeth 2008; 14: 206-11. Disponible en: http://www.scielo.cl [Consultado el 3 diciembre 2010].

2. Montoya C. Países que se desvían de la asociación habitual entre el nivel económico y el nivel de salud: algunos factores. Cuaderno Médico Sociales 2008; 48: 226-39.

3. Agostini C. Hacia un mapa de la desigualdad comunal en Chile. Perspectivas 2008; 17: 1-8. Disponible en: http://www.economiaynegocios.uahurtado.cl/ [Consultado el 22 noviembre 2010].

4. Urzúa A. Calidad de vida en salud relacionada con la salud: Elementos conceptuales. Rev Med Chile 2010; 138: 358-65.

5. Irwin L, Sidigi A, Hertzman C. Desarrollo de la primera infancia: un potente ecualizador. Informe final de Early Child Development Knowledge Network 2007. Disponible en: http://www.who.int/ [Consultado el 21 agosto 2010].

6. Consejo Asesor Presidencial. Hacia un Chile más justo. Trabajo, salario, competitividad y equidad social. Resumen ejecutivo del Consejo Asesor Presidencial 2008. Disponible en http://www.trabajoyequidad.cl/ [Consultado el 1 septiembre 2009].

7. Bredegal P, González P, Kotliarenco MA, Raczynski D. Chile: lineamientos de política para la primera infancia (menor de 4 años). Banco Interamericano de Desarrollo y UNICEF 2007. Disponible en: http://www.unicef.cl/ [Consultado el 1 septiembre 2009].

8. MIDEPLAN. Encuesta CASEN 2009. Disponible en: http://www.mideplan.cl/ [Consultado el 6 diciembre 2010].

9. Feito L. Vulnerabilidad. An Sist Sanit. Navar 2007; 30: 7-22.

10. Bertolozzi MR, Nichiata L, Ferreira R, Ciosak S, Hino P, Ferreira L et al. Os conceitos de vulnerabilidades e adesão na saúde colectiva. Rev Esc Enferm USP 2009; 43:
1326-30. Disponible. en http://www.ee.usp.br/ [Consultado el 21 septiembre 2010].

11. MIDEPLAN. Apuntes de protección Social. Publicación del Ministerio de Planificación, República de Chile, 2006; 2:1-4. Disponible en: http://www.mideplan.cl/. [Consultado el 5 enero 2010].

12. Marandola E, Hogan DJ. Vulnerabilidade do lugar vs Vulnerabilidade sociodemográfica: implicações metodológicas de uma velha questão. Rev bras Est Pop 2009; 26: 161-81. Disponible en: http://www.scielo.br/ [Consultado el 8 enero 2010].

13. Magalhães MH. O desafio da política de atendimento à infância e à adolescência na construção de políticas públicas eqüitativas. Cad. Saúde Pública 2002; 18 (Supl): 113-20.

14. OMS Subsanar las desigualdades en una generación. Alcanzar la equidad sanitaria actuando sobre los determinantes sociales de la salud, Organización mundial de la Salud 2008. Disponible en: http://www.who.int/ [Consultado el 21 enero 2010].

15. Mustard J. Early Human Development. Equity from the Start-Latin America. Rev latinoam cienc soc niñez juv 2009; 7: 639-680. Disponible en: http://www.umanizales. edu.co/ [Consultado el 21 julio 2010].

16. Arcos E, Uarac M, Molina I. Impacto de la violencia doméstica en la salud infantil. Rev Med Chile 2003; 131: 1454-62.

17. Talge N, Neal Ch, Glover V. Antenatal maternal stress and long-term effects on child neurodevelopment: how and why? J Child Psychology and Psychiatry 2007; 48: 3/4: 245-61.

18. Lazinski M, Shea A, Steiner M. Effects of maternal prenatal stress on offspring development: a commentary. Arch Womens Ment Health 2008; 11: 363-75.

19. Dunn S, Davies B, McCleary L, Edwards N, Gaboury S. The Relationship Between Vulnerability Factors and Breastfeeding Outcome. JOGNN Clinical Research 2006; 87-97.

20. Assis S, Quintes J, Vasconcellos R. Desigualdades socioeconómicas e saúde mental infantil. Rev Saúde Pública 2009; 43: 92-100.

21. PREDES. Protección social y primera infancia. Serie de documentos Predes, Facultad de Ciencias Sociales, Universidad de Chile, 2007. Disponible en: http://siis. mideplan.cl/ [Consultado el 15 marzo 2009].

22. Valenzuela MT. Indicadores socioeconómicos: relación con indicadores de salud. Ciencia \& Trabajo 2006; 19: 37-40. Disponible en: http:// www.cienciaytrabajo.cl. [Consultado el 15 octubre 2010].

23. Sunkel G. El papel de la familia en la protección social. Serie de Políticas Sociales 120, CEPAL 2006. Consulta- 
do en junio 2009. Disponible en: http://www.eclac.cl/ . [Consultado el 25 septiembre 2010].

24. Stevens G, Mascarenhas M, Mathers C. Global health risks: progress and challenges. Bull World Health Organ 2009; 87: 646. Disponible en: http://www.scielosp.org/ [Consultado el 5 octubre 2010].

25. Sojo A. Hacia la universalidad con solidaridad y eficiencia: el financiamiento de la protección social en países pobres y desiguales. Serie Seminarios y Conferencias 55, CEPAL 2009. Disponible en: http://www.eclac.org/ [Consultado el 25 septiembre 2010].

26. Caamaño E. Mujer y trabajo: origen y ocaso del modelo del padre proveedor y la madre cuidadora. Revista de Derecho de la Pontificia Universidad Católica de Valparaíso, 2010; 34. Disponible en http://www.scielo.cl/ [Consultado el 3 de octubre 2010].

27. Bellato R, Rocha W. Derechos y vulnebaribilidad:Nociones para ser exploradas para un abordaje ético en enfermería. Texto Contexto Enferm 2005; 14: 17-24. Disponible en: http://www.scielo.br/ [Consultado el 8 octubre 2010].

28. Gómez S, Muñoz, MM, Santelices MP. Efectividad de las Intervenciones en Apego con Infancia Vulnerada y en Riesgo Social: Un Desafío Prioritario para Chile. Ter Psicol 2008; 26: 241-251. Disponible en http://www. scielo.cl [Consultado el 5 octubre 2010].

29. Rodrigo MJ, Martín JC, Cabrera E, Máiquez ML. Las Competencias Parentales en Contextos de Riesgo Psicosocial. Intervención Psicosocial. 2009; 18: 113-20. Disponible en: http://scielo.isciii.es/ [Consultado el 10 octubre 2010].

30. Giovannella L, Vaistman J, Escorel S, Magalhães R, Costa N. Health and inequality: institutions and public policies in the 21st century. Rev Panam Salud Publica/Pan Am J Public Health 2002; 466-70. Disponible en: http://www. scielosp.org/ [Consultado el 30 octubre 2010].

31. Borrell C, Artazcoz L. Las políticas para medir las desigualdades en salud. Gac Sanit 2008; 22: 465-73. Disponible en: http://www.scielosp.org/ [Consultado el 30 octubre 2010].

32. Ryvadeneyra, Artundo C. La evaluación del impacto en salud: el estado de la cuestión. Gac Sanit 2008; 22:11-23. Disponible en http://scielo.isciii.es/ [Consultado el 5 de noviembre 2010].

33. OMS. La atención primaria en salud más necesaria que nunca. Organización Mundial de la Salud 2008. Disponible en: http://www.who.int [Consultado el 5 de noviembre 2010].

34. Laperriére H. Community health nursing practices in contexts of poverty, uncertaintyand unpredictability: a systematization of personal experiences. Rev Latino-am Enfermagem 2007; 15: 721-728. Disponible en: http:// www.scielo.br/ [Consultado el 2010].

35. Mulholland EK, Smith L, Carneiro I, Becher H, Lehmann D. Equity and child-survival strategies. Bull World Health Organ 2008; 86: 399-407 . Disponible en: http://www. scielosp.org/ [Consultado el 23 de noviembre 2010].

36. Franco-Giraldo A, Álvarez-Dardet C. Derechos humanos, una oportunidad para las políticas públicas en salud. Gac Sanit 2008; 22: 280-86. Disponible en: http:// www.scielosp.org/pdf/gs/v22n3/politicas.pdf [Consultado el 23 de noviembre 2010].

37. Calvete A, Campos P, Catalán D, Fernández K, Herrador $\mathrm{Z}$, Merino B, et al. Innovando en salud pública: monitorización de los determinantes sociales de la salud y reducción de las desigualdades en salud. Una prioridad para la presidencia española de la Unión Europea en 2010. Rev Esp Salud Pública 2010; 84: 3-11. Disponible en: http://redalyc.uaemex.mx [Consultado el 23 de noviembre 2010].

38. Villar E. Los Determinantes Sociales de Salud y la lucha por la equidad en Salud: desafíos para el Estado y la sociedad civil. Saúde Soc. São Paulo 2007; 16: 7-13. Disponible en: http://dds-dispositivoglobal.ops.org.ar/ [Consultado el 27 de noviembre 2010].

39. Sandín-Vásquez M, Sarría-Santamera A. Evaluación de impacto en salud: valorando la efectividad de las políticas en la salud de las poblaciones. Rev Esp Salud Pública 2008; 82: 261-72. Disponible en: http://scielo.isciii.es/ [Consultado el 27 de noviembre 2010].

40. Bermudez J, Mirosevic C. El acceso a la información pública como base para el control social y la protección del patrimonio público. Revista de Derecho de la Pontificia Universidad Católica de Valparaíso 2008; 31: 439-68. Disponible en: http://www.scielo.cl/ [Consultado el 20 septiembre 2010]. 